\title{
Dirac equation as a special case of Cosserat elasticity
}

\author{
James Burnett, Olga Chervova and Dmitri Vassiliev
}

\begin{abstract}
We suggest an alternative mathematical model for the electron in which the dynamical variables are a coframe (field of orthonormal bases) and a density. The electron mass and external electromagnetic field are incorporated into our model by means of a Kaluza-Klein extension. Our Lagrangian density is proportional to axial torsion squared. The advantage of our approach is that it does not require the use of spinors, Pauli matrices or covariant differentiation. The only geometric concepts we use are those of a metric, differential form, wedge product and exterior derivative. We prove that in the special case with no dependence on the third spatial coordinate our model is equivalent to the Dirac equation. The crucial element of the proof is the observation that our Lagrangian admits a factorisation.
\end{abstract}

Mathematics Subject Classification (2000). Primary 83E15; Secondary 53Z05.

Keywords. Dirac equation, Kaluza-Klein, spin, torsion.

\section{Introduction}

The Dirac equation is a system of four homogeneous linear complex partial differential equations for four complex unknowns. The unknowns (components of a bispinor) are functions of time and the three spatial coordinates. The Dirac equation is the accepted mathematical model for an electron and its antiparticle, the positron, in a given external electromagnetic field. One of the main applications of the Dirac equation is spectral-theoretic: it determines with high accuracy the energy levels of the hydrogen atom.

The geometric interpretation of the Dirac equation is rather complicated. It relies on the use of notions such as

- spinor,

- Pauli matrices,

- covariant derivative (note that formula (2.1) for the covariant derivative of a spinor field is quite tricky). 
There is also a logical problem with the Dirac equation in that distinguishing the electron from the positron forces one to resort to the concept of negative energy. Finally, the electromagnetic field is incorporated into the Dirac equation by means of a formal substitution which does not admit a simple geometric interpretation.

The purpose of this paper is to formulate an alternative mathematical model for the electron and positron, a model which is geometrically much simpler. The advantage of our approach is that it does not require the use of spinors, Pauli matrices or covariant differentiation. The only geometric concepts we use are those of a

- metric,

- differential form,

- wedge product,

- exterior derivative.

Our model overcomes the logical problem of distinguishing the electron from the positron: these correspond to clockwise and anticlockwise rotations of the coframe. And the electromagnetic field is incorporated into our model by means of a KaluzaKlein extension which has a simple geometric interpretation.

The paper has the following structure. In Section 2 we introduce our notation and in Section 3 we formulate the Dirac equation. In Section 4 we formulate our mathematical model and in Section 5 we translate our model into the language of bispinors. In Section 6 we prove Theorem 6.2 which is the main result of the paper: this theorem establishes that in the special case with no dependence on $x^{3}$ our mathematical model is equivalent to the Dirac equation. The crucial element of the proof of Theorem 6.2 is the observation that our Lagrangian admits a factorisation; this factorisation is the subject of Lemma 6.1. The concluding discussion is contained in Section $[7$

\section{Notation and conventions}

Throughout this paper we work on a 4-manifold $M$ equipped with prescribed Lorentzian metric $g$. All constructions presented in the paper are local so we do not make a priori assumptions on the geometric structure of spacetime $\{M, g\}$. The metric $g$ is not necessarily the Minkowski metric.

Our notation follows [1, 2]. In particular, in line with the traditions of particle physics, we use Greek letters to denote tensor (holonomic) indices.

By $\nabla$ we denote the covariant derivative with respect to the Levi-Civita connection. It acts on a vector field and a spinor field as $\nabla_{\alpha} v^{\beta}:=\partial_{\alpha} v^{\beta}+\Gamma^{\beta}{ }_{\alpha \gamma} v^{\gamma}$ and

$$
\nabla_{\alpha} \xi^{a}:=\partial_{\alpha} \xi^{a}+\frac{1}{4} \sigma_{\beta}^{a \dot{c}}\left(\partial_{\alpha} \sigma_{b \dot{c}}^{\beta}+\Gamma^{\beta}{ }_{\alpha \gamma} \sigma_{b \dot{c}}^{\gamma}\right) \xi^{b}
$$

respectively, where $\Gamma_{\alpha \gamma}^{\beta}=\left\{\begin{array}{c}\beta \\ \alpha \gamma\end{array}\right\}:=\frac{1}{2} g^{\beta \delta}\left(\partial_{\alpha} g_{\gamma \delta}+\partial_{\gamma} g_{\alpha \delta}-\partial_{\delta} g_{\alpha \gamma}\right)$ are the Christoffel symbols and $\sigma_{\beta}$ are Pauli matrices. 
We identify differential forms with covariant antisymmetric tensors. Given a pair of real covariant antisymmetric tensors $P$ and $Q$ of rank $r$ we define their dot product as $P \cdot Q:=\frac{1}{r !} P_{\alpha_{1} \ldots \alpha_{r}} Q_{\beta_{1} \ldots \beta_{r}} g^{\alpha_{1} \beta_{1}} \ldots g^{\alpha_{r} \beta_{r}}$. We also define $\|P\|^{2}:=P \cdot P$.

\section{The Dirac equation}

The following system of linear partial differential equations on $M$ is known as the Dirac equation:

$$
\sigma^{\alpha a \dot{b}}(i \nabla+A)_{\alpha} \eta_{\dot{b}}=m \xi^{a}, \quad \sigma_{a \dot{b}}^{\alpha}(i \nabla+A)_{\alpha} \xi^{a}=m \eta_{\dot{b}}
$$

Here $\xi^{a}, \eta_{\dot{b}}$ is a bispinor field which plays the role of dynamical variable (unknown quantity), $m$ is the electron mass and $A$ is the prescribed electromagnetic covector potential. The corresponding Lagrangian density is

$$
\begin{aligned}
L_{\text {Dir }}(\xi, \eta):= & \frac{i}{2}\left(\bar{\xi}^{\dot{b}} \sigma_{a \dot{b}}^{\alpha} \nabla_{\alpha} \xi^{a}-\xi^{a} \sigma_{a \dot{b}}^{\alpha} \nabla_{\alpha} \bar{\xi}^{\dot{b}}+\bar{\eta}_{a} \sigma^{\alpha a \dot{b}} \nabla_{\alpha} \eta_{\dot{b}}-\eta_{\dot{b}} \sigma^{\alpha a \dot{b}} \nabla_{\alpha} \bar{\eta}_{a}\right) \\
& \left.+A_{\alpha}\left(\xi^{a} \sigma_{a \dot{b}}^{\alpha} \bar{\xi}^{\dot{b}}+\bar{\eta}_{a} \sigma^{\alpha a \dot{b}} \eta_{\dot{b}}\right)-m\left(\xi^{a} \bar{\eta}_{a}+\bar{\xi}^{\dot{b}} \eta_{\dot{b}}\right)\right] \sqrt{|\operatorname{det} g|}
\end{aligned}
$$

\section{Our model}

A coframe $\vartheta$ is a quartet of real covector fields $\vartheta^{j}, j=0,1,2,3$, satisfying the constraint

$$
g=\vartheta^{0} \otimes \vartheta^{0}-\vartheta^{1} \otimes \vartheta^{1}-\vartheta^{2} \otimes \vartheta^{2}-\vartheta^{3} \otimes \vartheta^{3} .
$$

For the sake of clarity we repeat formula (4.1) giving the tensor indices explicitly: $g_{\alpha \beta}=\vartheta_{\alpha}^{0} \vartheta_{\beta}^{0}-\vartheta_{\alpha}^{1} \vartheta_{\beta}^{1}-\vartheta_{\alpha}^{2} \vartheta_{\beta}^{2}-\vartheta_{\alpha}^{3} \vartheta_{\beta}^{3}$.

Formula (4.1) means that the coframe is a field of orthonormal bases with orthonormality understood in the Lorentzian sense. Of course, at every point of the manifold $M$ the choice of coframe is not unique: there are 6 real degrees of freedom in choosing the coframe and any pair of coframes is related by a Lorentz transformation.

As dynamical variables in our model we choose a coframe $\vartheta$ and a positive density $\rho$. These live in the original $(1+3)$-dimensional spacetime $\{M, g\}$ and are functions of local coordinates $\left(x^{0}, x^{1}, x^{2}, x^{3}\right)$.

In order to incorporate into our model mass and electromagnetic field we perform a Kaluza-Klein extension: we add an extra coordinate $x^{4}$ and work on the resulting 5 -manifold which we denote by $\mathbf{M}$. We suppose that

- the coordinate $x^{4}$ is fixed,

- we allow only changes of coordinates $\left(x^{0}, x^{1}, x^{2}, x^{3}\right)$ which do not depend on $x^{4}$.

We will use bold type for extended quantities.

We extend our coframe as

$$
\boldsymbol{\vartheta}_{\boldsymbol{\alpha}}^{0}=\left(\begin{array}{c}
\vartheta_{\alpha}^{0} \\
0
\end{array}\right), \quad \boldsymbol{\vartheta}_{\boldsymbol{\alpha}}^{3}=\left(\begin{array}{c}
\vartheta_{\alpha}^{3} \\
0
\end{array}\right)
$$




$$
\begin{gathered}
\left(\boldsymbol{\vartheta}^{1}+i \boldsymbol{\vartheta}^{2}\right)_{\boldsymbol{\alpha}}=\left(\begin{array}{c}
\left(\vartheta^{1}+i \vartheta^{2}\right)_{\alpha} \\
0
\end{array}\right) e^{-2 i m x^{4}}, \\
\boldsymbol{\vartheta}_{\boldsymbol{\alpha}}^{4}=\left(\begin{array}{c}
0_{\alpha} \\
1
\end{array}\right)
\end{gathered}
$$

where the bold tensor index $\boldsymbol{\alpha}$ runs through the values $0,1,2,3$, 4, whereas its non-bold counterpart $\alpha$ runs through the values $0,1,2,3$. In particular, the $0_{\alpha}$ in formula (4.4) stands for a column of four zeros.

The coordinate $x^{4}$ parametrises a circle of radius $\frac{1}{2 m}$. Condition (4.3) means that the extended coframe $\boldsymbol{\vartheta}$ experiences a full turn in the $\left(\vartheta^{1}, \vartheta^{2}\right)$-plane as we move along this circle, coming back to the starting point.

We extend our metric as

$$
\mathbf{g}_{\boldsymbol{\alpha} \boldsymbol{\beta}}:=\left(\begin{array}{cc}
g_{\alpha \beta}-\frac{1}{m^{2}} A_{\alpha} A_{\beta} & \frac{1}{m} A_{\alpha} \\
\frac{1}{m} A_{\beta} & -1
\end{array}\right) .
$$

Formula (4.5) means that we view electromagnetism as a perturbation (shear) of the extended metric. Recall that in classical elasticity "shear" stands for "perturbation of the metric which does not change the volume". It is easy to see that formula (4.5) implies $\operatorname{det} \mathbf{g}=-\operatorname{det} g$, so $\operatorname{det} \mathbf{g} \operatorname{does} n o t$ depend on $A$ and, hence, the electromagnetic field does not change the volume form.

Note that when $A \neq 0$ the extended coframe and the extended metric no longer agree:

$$
\mathrm{g} \neq \boldsymbol{\vartheta}^{0} \otimes \boldsymbol{\vartheta}^{0}-\boldsymbol{\vartheta}^{1} \otimes \boldsymbol{\vartheta}^{1}-\boldsymbol{\vartheta}^{2} \otimes \boldsymbol{\vartheta}^{2}-\boldsymbol{\vartheta}^{3} \otimes \boldsymbol{\vartheta}^{3}-\boldsymbol{\vartheta}^{4} \otimes \boldsymbol{\vartheta}^{4}
$$

(compare with (4.1)). The full physical implications of this discord are not discussed in the current paper. We need the extended metric only for raising tensor indices (see formula (4.9) below) and for this purpose the discord (4.6) is irrelevant.

We define the 3 -form

$$
\mathbf{T}^{\text {ax }}:=\frac{1}{3}(\boldsymbol{\vartheta}^{0} \wedge d \boldsymbol{\vartheta}^{0}-\boldsymbol{\vartheta}^{1} \wedge d \boldsymbol{\vartheta}^{1}-\boldsymbol{\vartheta}^{2} \wedge d \boldsymbol{\vartheta}^{2}-\boldsymbol{\vartheta}^{3} \wedge d \boldsymbol{\vartheta}^{3}-\underbrace{\boldsymbol{\vartheta}^{4} \wedge d \boldsymbol{\vartheta}^{4}}_{=0})
$$

where $d$ denotes the exterior derivative. This 3-form is called axial torsion of the teleparallel connection. An explanation of the geometric meaning of the latter phrase as well as a detailed exposition of the application of torsion in field theory and the history of the subject can be found in 3 . For our purposes the 3 -form (4.7) is simply a measure of deformations generated by rotations of spacetime points.

We choose our Lagrangian density to be

$$
L(\vartheta, \rho):=\left\|\mathbf{T}^{\mathrm{ax}}\right\|^{2} \rho
$$

where

$$
\left\|\mathbf{T}^{\mathrm{ax}}\right\|^{2}:=\frac{1}{3 !} \mathbf{T}_{\boldsymbol{\alpha} \boldsymbol{\beta} \gamma}^{\mathrm{ax}} \mathbf{T}_{\kappa \lambda \mu}^{\mathrm{ax}} \mathrm{g}^{\alpha \kappa} \mathrm{g}^{\boldsymbol{\beta} \boldsymbol{\lambda}} \mathrm{g}^{\gamma \mu}
$$

Formula (4.3) implies

$$
\boldsymbol{\vartheta}^{1} \wedge d \boldsymbol{\vartheta}^{1}+\boldsymbol{\vartheta}^{2} \wedge d \boldsymbol{\vartheta}^{2}=\vartheta^{1} \wedge d \vartheta^{1}+\vartheta^{2} \wedge d \vartheta^{2}-4 m \vartheta^{1} \wedge \vartheta^{2} \wedge \boldsymbol{\vartheta}^{4}
$$


so our Lagrangian density $L(\vartheta, \rho)$ does not depend on $x^{4}$ and can be viewed as a Lagrangian density in the original spacetime of dimension $1+3$. This means, essentially, that we have performed a separation of variables in a nonlinear setting.

Our action (variational functional) is $\int L(\vartheta, \rho) d x^{0} d x^{1} d x^{2} d x^{3}$. Our field equations (Euler-Lagrange equations) are obtained by varying this action with respect to the coframe $\vartheta$ and density $\rho$. Varying with respect to the density $\rho$ is easy: this gives the field equation $\left\|\mathbf{T}^{\mathrm{ax}}\right\|^{2}=0$ which is equivalent to $L(\vartheta, \rho)=0$. Varying with respect to the coframe $\vartheta$ is more difficult because we have to maintain the metric constraint (4.1); recall that the metric is assumed to be prescribed (fixed).

We do not write down the field equations for the Lagrangian density $L(\vartheta, \rho)$ explicitly. We note only that they are highly nonlinear and do not appear to bear any resemblance to the linear Dirac equation (3.1).

\section{Choosing a common language}

In order to compare the two models described in Sections 3 and 4 we need to choose a common mathematical language. We choose the language of bispinors. Namely, we express the coframe and density via a bispinor field according to formulae

$$
\begin{gathered}
s=\xi^{a} \bar{\eta}_{a}, \\
\rho=|s| \sqrt{\operatorname{det}\left|g_{\alpha \beta}\right|}, \\
\left(\vartheta^{0}+\vartheta^{3}\right)_{\alpha}=|s|^{-1} \xi^{a} \sigma_{\alpha a \dot{b}} \bar{\xi}^{\dot{b}} \\
\left(\vartheta^{0}-\vartheta^{3}\right)_{\alpha}=|s|^{-1} \bar{\eta}^{a} \sigma_{\alpha a \dot{b}} \eta^{\dot{b}} \\
\left(\vartheta^{1}+i \vartheta^{2}\right)_{\alpha}=-|s|^{-1} \xi^{a} \sigma_{\alpha a \dot{b}} \eta^{\dot{b}}
\end{gathered}
$$

where

$$
\eta^{\dot{a}}=\epsilon^{\dot{a} \dot{b}} \eta_{\dot{b}}, \quad \epsilon_{a b}=\epsilon_{\dot{a} \dot{b}}=\epsilon^{a b}=\epsilon^{\dot{a} \dot{b}}=\left(\begin{array}{cc}
0 & 1 \\
-1 & 0
\end{array}\right) .
$$

Note that throughout this paper we assume that the density $\rho$ does not vanish.

Observe now that the right-hand sides of formulae (5.2)-(5.5) are invariant under the change of bispinor field $\xi^{a} \mapsto \xi^{a} e^{i \varphi}, \eta_{\dot{b}} \mapsto \eta_{\dot{b}} e^{-i \varphi}$ where $\varphi: M \rightarrow \mathbb{R}$ is an arbitrary scalar function. In other words, formulae (5.2)-(5.5) do not feel the argument of the complex scalar $s$. Hence, when translating our model into the language of bispinors it is natural to impose the constraint

$$
\operatorname{Im} s=0, \quad s>0 .
$$

This constraint reflects the fact that our model has one real dynamical degree of freedom less than the Dirac model (seven real degrees of freedom instead of eight). 


\section{Special case with no dependence on $x^{3}$}

In addition to our usual assumptions (see beginning of Section 4) we suppose that

- the coordinate $x^{3}$ is fixed,

- we allow only changes of coordinates $\left(x^{0}, x^{1}, x^{2}\right)$ which do not depend on $x^{3}$,

- the metric does not depend on $x^{3}$ and has block structure

$$
g_{\alpha \beta}=\left(\begin{array}{cccc}
g_{00} & g_{01} & g_{02} & 0 \\
g_{10} & g_{11} & g_{12} & 0 \\
g_{20} & g_{21} & g_{22} & 0 \\
0 & 0 & 0 & -1
\end{array}\right),
$$

- the electromagnetic covector potential does not depend on $x^{3}$ and has $A_{3}=0$.

We work with coframes such that

$$
\vartheta_{\alpha}^{3}=\left(\begin{array}{l}
0 \\
0 \\
0 \\
1
\end{array}\right)
$$

We use Pauli matrices which do not depend on $x^{3}$ and take

$$
\sigma_{3 a b}=\left(\begin{array}{cc}
1 & 0 \\
0 & -1
\end{array}\right) \text {. }
$$

We take

$$
\eta_{\dot{b}}=\xi^{a} \sigma_{3 a \dot{b}} .
$$

Then the scalar defined by formula (5.1) takes the form $s=\left|\xi^{1}\right|^{2}-\left|\xi^{2}\right|^{2}$. This scalar is automatically real and condition (5.7) becomes

$$
\left|\xi^{1}\right|^{2}-\left|\xi^{2}\right|^{2}>0
$$

It is easy to see that formulae (6.1), (6.3)- 6.5 imply 6.2

Formula (6.4) means that our bispinor $\xi^{a}, \eta_{\dot{b}}$ is determined by the spinor $\xi^{a}$. Thus, the spinor $\xi^{a}$ becomes the (only) dynamical variable. We assume that this spinor does not depend on $x^{3}$.

Observe that in the special case considered in this section both the Dirac model and our model have the same number of real dynamical degrees of freedom, namely, four. This is because under the assumption (6.2) the coframe $\vartheta$ and density $\rho$ are equivalent to a spinor field $\xi^{a}$ modulo sign $\left(-\xi^{a}\right.$ gives the same $\vartheta$ and $\left.\rho\right)$.

Throughout this section summation is carried out either over indices $0,1,2$ or over indices $0,1,2,4$. In the latter case we use bold type.

Put

$$
\begin{aligned}
L_{\mathrm{Dir}}^{ \pm}(\xi):=\left[\frac{i}{2}\left(\bar{\xi}^{\dot{b}} \sigma^{\alpha}{ }_{a \dot{b}} \nabla_{\alpha} \xi^{a}-\xi^{a} \sigma^{\alpha}{ }_{a \dot{b}} \nabla_{\alpha} \bar{\xi}^{\dot{b}}\right)\right. & \\
& \left.+A_{\alpha} \xi^{a} \sigma_{a \dot{b}}^{\alpha} \bar{\xi}^{\dot{b}} \mp m \xi^{a} \sigma_{3 a \dot{b}} \bar{\xi}^{\dot{b}}\right] \sqrt{|\operatorname{det} g|}
\end{aligned}
$$

The Lagrangian densities $L_{\mathrm{Dir}}^{ \pm}(\xi)$ are formally related to the original Lagrangian density (3.2) as follows: if we set $\eta_{\dot{b}}= \pm \xi^{a} \sigma_{3 a \dot{b}}$ we get $L_{\operatorname{Dir}}(\xi, \eta)=2 L_{\text {Dir }}^{ \pm}(\xi)$. We say 
"formally related" because in this section we assume that formula $\eta_{\dot{b}}= \pm \xi^{a} \sigma_{3 a \dot{b}}$ holds with upper sign, see (6.4). The $L_{\text {Dir }}^{+}(\xi)$ and $L_{\text {Dir }}^{-}(\xi)$ are, of course, the usual Dirac Lagrangian densities for an electron with spin up and spin down.

Lemma 6.1. In the special case with no dependence on $x^{3}$ our Lagrangian density (4.8) factorises as

$$
L(\vartheta, \rho)=-\frac{32 m}{9} \frac{L_{\text {Dir }}^{+}(\xi) L_{\text {Dir }}^{-}(\xi)}{L_{\text {Dir }}^{+}(\xi)-L_{\text {Dir }}^{-}(\xi)} .
$$

Let us emphasise once again that throughout this paper we assume that the density $\rho$ does not vanish. In the special case with no dependence on $x^{3}$ this assumption can be equivalently rewritten as

$$
L_{\text {Dir }}^{+}(\xi) \neq L_{\text {Dir }}^{-}(\xi)
$$

so the denominator in (6.7) is nonzero.

Proof. Step 1. Let us show that it is sufficient to prove formula 6.7 under the assumption $d A=0$, i.e. under the assumption that the electromagnetic covector potential $A$ is pure gauge. Recall that $d A$ stands for the exterior derivative of $A$.

Suppose that we have already proved formula (6.7) under the assumption $d A=0$ and are now looking at the case of general $A$. Let us fix an arbitrary point $P$ on our 4-manifold $M$ and prove formula (6.7) at this point. To do this, we perturb the electromagnetic covector potential $A$ in such a way that

- $A$ retains its value at the point $P$ and

- $A$ satisfies the condition $d A=0$ in a neighbourhood of $P$.

This can be achieved by, say, choosing some local coordinates on $M$ and setting the components of $A$ to be constant in this coordinate system. Now, this perturbation of the covector potential $A$ does not change the LHS or the RHS of (6.7) at the point $P$ because neither of them depends on derivatives of $A$. Hence, the case of general $A$ has been reduced to the case $d A=0$.

Step 2. Let us show that it is sufficient to prove formula (6.7) under the assumption $A=0$.

Suppose that we have already proved formula (6.7) under the assumption $A=0$ and are now looking at the case $d A=0$. Let us modify the definition of the extended coframe by replacing (4.4) with

$$
\boldsymbol{\vartheta}_{\boldsymbol{\alpha}}^{4}=\left(\begin{array}{c}
-\frac{1}{m} A_{\alpha} \\
1
\end{array}\right)
$$

In view of the condition $d A=0$ this modification of the extended coframe does not change axial torsion (4.7) but the extended coframe (4.2), (4.3), (6.9) now agrees with the extended metric (4.5): we have

$$
\mathrm{g}=\boldsymbol{\vartheta}^{0} \otimes \boldsymbol{\vartheta}^{0}-\boldsymbol{\vartheta}^{1} \otimes \boldsymbol{\vartheta}^{1}-\boldsymbol{\vartheta}^{2} \otimes \boldsymbol{\vartheta}^{2}-\boldsymbol{\vartheta}^{3} \otimes \boldsymbol{\vartheta}^{3}-\boldsymbol{\vartheta}^{4} \otimes \boldsymbol{\vartheta}^{4}
$$


as opposed to (4.6). Let us now perform a change of coordinates

$$
\tilde{x}^{\alpha}=x^{\alpha}, \quad \alpha=0,1,2,3, \quad \tilde{x}^{4}=x^{4}-\frac{1}{m} \int A \cdot d x .
$$

Note that the integral $\int A \cdot d x$ is (locally) well-defined because of the assumption $d A=0$. The change of coordinates (6.11) is against the rules we stated in the beginning of Section 4 when describing our model (we changed the original Kaluza coordinate $x^{4}$ to a new coordinate $\tilde{x}^{4}$ ) but we are doing this only for the purpose of proving the lemma. In the new coordinate system $\tilde{x}$ the extended coframe (4.2), (4.3), (6.9) takes its original form (4.2)-(4.4), the extended metric takes the form $\mathbf{g}_{\boldsymbol{\alpha} \boldsymbol{\beta}}=\left(\begin{array}{cc}g_{\alpha \beta} & 0 \\ 0 & -1\end{array}\right)$ (compare with (4.5) $)$ and the electromagnetic covector potential $A$ is not affected (i.e. it has the same components in both coordinate systems). Observe now that in (4.3) we have retained the scalar factor $e^{-2 i m x^{4}}$ written in terms of the original Kaluza coordinate $x^{4}$. Expressing $x^{4}$ in terms of $\tilde{x}^{4}$ in accordance with formula (6.11) we get

$$
\left(\boldsymbol{\vartheta}^{1}+i \boldsymbol{\vartheta}^{2}\right)_{\boldsymbol{\alpha}}=\left(\begin{array}{c}
\left(\vartheta^{1}+i \vartheta^{2}\right)_{\alpha} \\
0
\end{array}\right) e^{-2 i m \tilde{x}^{4}-2 i \int A \cdot d x} .
$$

Let us now introduce a new coframe $\hat{\vartheta}$ in $(1+3)$-dimensional spacetime $\{M, g\}$ related to the original coframe $\vartheta$ as

$$
\hat{\vartheta}^{0}=\vartheta^{0}, \quad \hat{\vartheta}^{3}=\vartheta^{3}, \quad \hat{\vartheta}^{1}+i \hat{\vartheta}^{2}=\left(\vartheta^{1}+i \vartheta^{2}\right) e^{-2 i \int A \cdot d x} .
$$

Then formulae (6.12), (6.13) imply

$$
L(\hat{\vartheta}, \rho ; 0)=L(\vartheta, \rho ; A) .
$$

Here $L(\cdot, \cdot ; \cdot)$ is our Lagrangian density $L(\cdot, \cdot)$ defined by formulae (4.2)-4.5], (4.7) -(4.9) but with an extra entry after the semicolon for the electromagnetic covector potential. Formula (6.14) means that in our model the introduction of an electromagnetic covector potential $A$ satisfying the condition $d A=0$ is equivalent to a change of coframe (6.13).

Formulae (5.1)-(5.6), (6.4) imply that the change of coframe (6.13) leads to a change of spinor field $\hat{\xi}^{a}=\xi^{a} e^{-i \int A \cdot d x}$. Substituting the latter into (6.6) we get

$$
L_{\text {Dir }}^{ \pm}(\hat{\xi} ; 0)=L_{\text {Dir }}^{ \pm}(\xi ; A) .
$$

Here $L_{\text {Dir }}^{ \pm}(\cdot ; \cdot)$ is the Dirac Lagrangian density $L_{\text {Dir }}^{ \pm}(\cdot)$ defined by formula (6.6) but with an extra entry after the semicolon for the electromagnetic covector potential.

In the beginning of this part of the proof we assumed that we have already proved formula (6.7) under the assumption $A=0$ so we have

$$
L(\hat{\vartheta}, \rho ; 0)=-\frac{32 m}{9} \frac{L_{\text {Dir }}^{+}(\hat{\xi} ; 0) L_{\text {Dir }}^{-}(\hat{\xi} ; 0)}{L_{\text {Dir }}^{+}(\hat{\xi} ; 0)-L_{\text {Dir }}^{-}(\hat{\xi} ; 0)} .
$$


It remains to note that formulae (6.14) - 6.16) imply 6.7 . Hence, the case $d A=0$ has been reduced to the case $A=0$.

Step 3. In the remainder of the proof we assume that $A=0$.

The proof of formula (6.7) is performed by direct substitution: it is just a matter of expressing the coframe and density via the spinor using formulae (5.1)(5.6), (6.4) and substituting these expressions into the LHS of (6.7). However, even with $A=0$ this is a massive calculation. In order to overcome these technical difficulties we perform below a trick which makes the calculations much easier. This trick is a known one and was, for example, extensively used by A. Dimakis and F. Müller-Hoissen [4, 5, 6].

Observe that when working with spinors we have the freedom in our choice of Pauli matrices: at every point of our $(1+3)$-dimensional spacetime $\{M, g\}$ we can apply a proper Lorentz transformation to a given set of Pauli matrices to get a new set of Pauli matrices, with spinor fields transforming accordingly. It is sufficient to prove formula (6.7) for one particular choice of Pauli matrices, hence it is natural to choose Pauli matrices in a way that makes calculations as simple as possible. We choose Pauli matrices

$$
\sigma_{\alpha a \dot{b}}=\vartheta_{\alpha}^{j} s_{j a \dot{b}}=\vartheta_{\alpha}^{0} s_{0 a \dot{b}}+\vartheta_{\alpha}^{1} s_{1 a \dot{b}}+\vartheta_{\alpha}^{2} s_{2 a \dot{b}}+\vartheta_{\alpha}^{3} s_{3 a \dot{b}}
$$

where

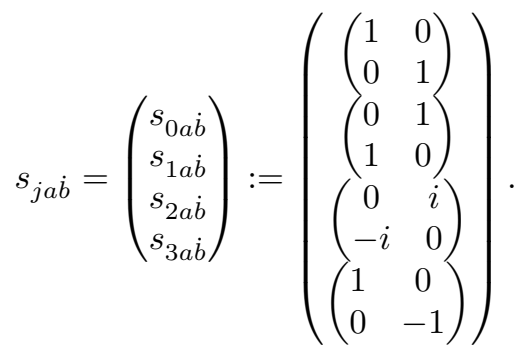

Here $\vartheta$ is the coframe that appears in the LHS of formula (6.7). Let us stress that in the statement of the lemma Pauli matrices are not assumed to be related in any way to the coframe $\vartheta$. We are just choosing the particular Pauli matrices (6.17), (6.18) to simplify calculations in our proof.

Examination of formulae (5.1)-(5.6), (6.4), (6.5), 6.17), (6.18) shows that with our special choice of Pauli matrices we have $\xi^{2}=0$ whereas $\xi^{1}$ is nonzero and real. We are about to write down the Dirac Lagrangian density (6.6) which is quadratic in $\xi$ so the sign of $\xi$ does not matter. So let $\xi^{a}=\left(\begin{array}{c}e^{h} \\ 0\end{array}\right)$ where $h: M \rightarrow \mathbb{R}$ 
is a scalar function. We get

$$
\begin{aligned}
& \frac{i}{2} \bar{\xi}^{\dot{d}} \sigma_{a \dot{d}}^{\alpha} \nabla_{\alpha} \xi^{a}=\frac{i}{2} \bar{\xi}^{\dot{d}}\left(\sigma_{a \dot{d}}^{\alpha} \partial_{\alpha} h\right) \xi^{a}+\frac{i}{8} \bar{\xi}^{\dot{d}} \sigma_{a \dot{d}}^{\alpha} \sigma_{\beta}{ }^{a \dot{c}}\left(\partial_{\alpha} \sigma_{b \dot{c}}^{\beta}+\Gamma^{\beta}{ }_{\alpha \gamma} \sigma_{b \dot{c}}^{\gamma}\right) \xi^{b} \\
& =\frac{i e^{2 h}}{8} \sigma_{a \dot{1}}^{\alpha} \sigma_{\beta}{ }^{a \dot{c}}\left(\partial_{\alpha} \sigma^{\beta}{ }_{1 \dot{c}}+\Gamma^{\beta}{ }_{\alpha \gamma} \sigma^{\gamma}{ }_{1 \dot{c}}\right)+\ldots=\frac{i e^{2 h}}{8} \sigma^{\alpha}{ }_{a \dot{1}} \sigma_{\beta}{ }^{a \dot{c}} \nabla_{\alpha} \sigma^{\beta}{ }_{1 \dot{c}}+\ldots \\
& =\frac{i e^{2 h}}{8}\left[\sigma^{\alpha}{ }_{1 \dot{1}} \sigma_{\beta}{ }^{1 \dot{1}} \nabla_{\alpha} \sigma^{\beta}{ }_{1 \dot{1}}+\sigma^{\alpha}{ }_{1 \dot{1}} \sigma_{\beta}{ }^{1 \dot{2}} \nabla_{\alpha} \sigma^{\beta}{ }_{1 \dot{2}}+\sigma^{\alpha}{ }_{2 \dot{1}} \sigma_{\beta}{ }^{2 \dot{1}} \nabla_{\alpha} \sigma^{\beta}{ }_{1 \dot{1}}+\sigma^{\alpha}{ }_{2 \dot{1}} \sigma_{\beta}{ }^{2 \dot{2}} \nabla_{\alpha} \sigma^{\beta}{ }_{1 \dot{2}}\right]+\ldots \\
& =\frac{i e^{2 h}}{8}\left[\vartheta^{0 \alpha} \sigma_{\beta}^{1 \dot{1}} \nabla_{\alpha} \sigma_{1 \dot{1}}^{\beta}+\vartheta^{0 \alpha} \sigma_{\beta}{ }^{1 \dot{2}} \nabla_{\alpha} \sigma_{1 \dot{2}}^{\beta}\right. \\
& \left.+\left(\vartheta^{1}-i \vartheta^{2}\right)^{\alpha} \sigma_{\beta}{ }^{2 \dot{1}} \nabla_{\alpha} \sigma_{1 \dot{1}}^{\beta}+\left(\vartheta^{1}-i \vartheta^{2}\right)^{\alpha} \sigma_{\beta}{ }^{2 \dot{2}} \nabla_{\alpha} \sigma_{1 \dot{2}}^{\beta}\right]+\ldots \\
& =\frac{i e^{2 h}}{8}\left[\vartheta^{0 \alpha} \sigma_{\beta}^{1 \dot{1}} \nabla_{\alpha} \vartheta^{0 \beta}+\vartheta^{0 \alpha} \sigma_{\beta}^{1 \dot{2}} \nabla_{\alpha}\left(\vartheta^{1}+i \vartheta^{2}\right)^{\beta}\right. \\
& \left.+\left(\vartheta^{1}-i \vartheta^{2}\right)^{\alpha} \sigma_{\beta}{ }^{2 i} \nabla_{\alpha} \vartheta^{0 \beta}+\left(\vartheta^{1}-i \vartheta^{2}\right)^{\alpha} \sigma_{\beta}{ }^{2 \dot{2}} \nabla_{\alpha}\left(\vartheta^{1}+i \vartheta^{2}\right)^{\beta}\right]+\ldots \\
& =\frac{i e^{2 h}}{8}\left[\vartheta^{0 \alpha} \vartheta_{\beta}^{0} \nabla_{\alpha} \vartheta^{0 \beta}-\vartheta^{0 \alpha}\left(\vartheta^{1}-i \vartheta^{2}\right)_{\beta} \nabla_{\alpha}\left(\vartheta^{1}+i \vartheta^{2}\right)^{\beta}\right. \\
& \left.-\left(\vartheta^{1}-i \vartheta^{2}\right)^{\alpha}\left(\vartheta^{1}+i \vartheta^{2}\right)_{\beta} \nabla_{\alpha} \vartheta^{0 \beta}+\left(\vartheta^{1}-i \vartheta^{2}\right)^{\alpha} \vartheta_{\beta}^{0} \nabla_{\alpha}\left(\vartheta^{1}+i \vartheta^{2}\right)^{\beta}\right]+\ldots \\
& =\frac{i e^{2 h}}{8}\left[-i \vartheta^{0 \alpha} \vartheta_{\beta}^{1} \nabla_{\alpha} \vartheta^{2 \beta}+i \vartheta^{0 \alpha} \vartheta_{\beta}^{2} \nabla_{\alpha} \vartheta^{1 \beta}-i \vartheta^{1 \alpha} \vartheta_{\beta}^{2} \nabla_{\alpha} \vartheta^{0 \beta}\right. \\
& \left.+i \vartheta^{2 \alpha} \vartheta_{\beta}^{1} \nabla_{\alpha} \vartheta^{0 \beta}+i \vartheta^{1 \alpha} \vartheta_{\beta}^{0} \nabla_{\alpha} \vartheta^{2 \beta}-i \vartheta^{2 \alpha} \vartheta_{\beta}^{0} \nabla_{\alpha} \vartheta^{1 \beta}\right]+\ldots \\
& =\frac{e^{2 h}}{8}\left[\vartheta^{0 \alpha} \vartheta_{\beta}^{1} \nabla_{\alpha} \vartheta^{2 \beta}-\vartheta^{0 \alpha} \vartheta_{\beta}^{2} \nabla_{\alpha} \vartheta^{1 \beta}+\vartheta^{1 \alpha} \vartheta_{\beta}^{2} \nabla_{\alpha} \vartheta^{0 \beta}\right. \\
& \left.-\vartheta^{2 \alpha} \vartheta_{\beta}^{1} \nabla_{\alpha} \vartheta^{0 \beta}-\vartheta^{1 \alpha} \vartheta_{\beta}^{0} \nabla_{\alpha} \vartheta^{2 \beta}+\vartheta^{2 \alpha} \vartheta_{\beta}^{0} \nabla_{\alpha} \vartheta^{1 \beta}\right]+\ldots \\
& =\frac{s}{8}\left[\left(\vartheta^{0} \wedge \vartheta^{1}\right) \cdot d \vartheta^{2}+\left(\vartheta^{1} \wedge \vartheta^{2}\right) \cdot d \vartheta^{0}+\left(\vartheta^{2} \wedge \vartheta^{0}\right) \cdot d \vartheta^{1}\right]+\ldots
\end{aligned}
$$

where the dots denote purely imaginary terms. Hence,

$$
\frac{i}{2}\left(\bar{\xi}^{\dot{b}} \sigma_{a \dot{b}}^{\alpha} \nabla_{\alpha} \xi^{a}-\xi^{a} \sigma_{a \dot{b}}^{\alpha} \nabla_{\alpha} \bar{\xi}^{\dot{b}}\right)=\frac{s}{4}\left[\left(\vartheta^{0} \wedge \vartheta^{1}\right) \cdot d \vartheta^{2}+\left(\vartheta^{1} \wedge \vartheta^{2}\right) \cdot d \vartheta^{0}+\left(\vartheta^{2} \wedge \vartheta^{0}\right) \cdot d \vartheta^{1}\right] .
$$

Formula (6.6) with $A=0$ can now be rewritten as

$$
L_{\mathrm{Dir}}^{ \pm}(\xi)=\left[\frac{1}{4}\left[\left(\vartheta^{0} \wedge \vartheta^{1}\right) \cdot d \vartheta^{2}+\left(\vartheta^{1} \wedge \vartheta^{2}\right) \cdot d \vartheta^{0}+\left(\vartheta^{2} \wedge \vartheta^{0}\right) \cdot d \vartheta^{1}\right] \mp m\right] \rho \text {. }
$$

Put

$$
T^{\mathrm{ax}}:=\frac{1}{3}(\vartheta^{0} \wedge d \vartheta^{0}-\vartheta^{1} \wedge d \vartheta^{1}-\vartheta^{2} \wedge d \vartheta^{2}-\underbrace{\vartheta^{3} \wedge d \vartheta^{3}}_{=0})
$$

(compare with (4.7)). The last term in (6.20) vanishes in view of (6.2). The coordinate $x^{3}$ is redundant so $T^{\mathrm{ax}}$ can be viewed as a 3 -form in $(1+2)$-dimensional Lorentzian space with local coordinates $\left(x^{0}, x^{1}, x^{2}\right)$. Hence, we can define the scalar

$$
* T^{\mathrm{ax}}:=\frac{1}{3 !} \sqrt{|\operatorname{det} g|}\left(T^{\mathrm{ax}}\right)^{\alpha \beta \gamma} \varepsilon_{\alpha \beta \gamma}
$$


which is the Hodge dual of $T^{\mathrm{ax}}$. But $\sqrt{|\operatorname{det} g|} \varepsilon_{\alpha \beta \gamma}=\left(\vartheta^{0} \wedge \vartheta^{1} \wedge \vartheta^{2}\right)_{\alpha \beta \gamma}$ so formula (6.21) can be rewritten as

$$
\begin{aligned}
* T^{\mathrm{ax}}=T^{\mathrm{ax}} \cdot\left(\vartheta^{0} \wedge \vartheta^{1}\right. & \left.\wedge \vartheta^{2}\right)=\frac{1}{3}\left(\vartheta^{0} \wedge d \vartheta^{0}-\vartheta^{1} \wedge d \vartheta^{1}-\vartheta^{2} \wedge d \vartheta^{2}\right) \cdot\left(\vartheta^{0} \wedge \vartheta^{1} \wedge \vartheta^{2}\right) \\
= & \frac{1}{3}\left[\left(\vartheta^{0} \wedge \vartheta^{1}\right) \cdot d \vartheta^{2}+\left(\vartheta^{1} \wedge \vartheta^{2}\right) \cdot d \vartheta^{0}+\left(\vartheta^{2} \wedge \vartheta^{0}\right) \cdot d \vartheta^{1}\right] .
\end{aligned}
$$

Substituting the latter into (6.19) we arrive at the compact formula

$$
L_{\text {Dir }}^{ \pm}(\xi)=\left[\frac{3}{4} * T^{\mathrm{ax}} \mp m\right] \rho .
$$

Substituting (6.22) into the RHS of (6.7) we get

$$
-\frac{32 m}{9} \frac{L_{\text {Dir }}^{+}(\xi) L_{\text {Dir }}^{-}(\xi)}{L_{\text {Dir }}^{+}(\xi)-L_{\text {Dir }}^{-}(\xi)}=\left[\left(* T^{\mathrm{ax}}\right)^{2}-\frac{16}{9} m^{2}\right] \rho .
$$

As our Lagrangian $L(\vartheta, \rho)$ is defined by formula (4.8), the proof of the lemma has been reduced to proving

$$
\left\|\mathbf{T}^{\mathrm{ax}}\right\|^{2}=\left(* T^{\mathrm{ax}}\right)^{2}-\frac{16}{9} m^{2}
$$

with $A=0$ (recall that $A$ initially appeared in the extended metric (4.5)).

In view of (4.2), (6.2) formula (4.7) becomes

$$
\mathbf{T}^{\mathrm{ax}}=\frac{1}{3}\left(\boldsymbol{\vartheta}^{0} \wedge d \boldsymbol{\vartheta}^{0}-\boldsymbol{\vartheta}^{1} \wedge d \boldsymbol{\vartheta}^{1}-\boldsymbol{\vartheta}^{2} \wedge d \boldsymbol{\vartheta}^{2}\right) .
$$

The difference between formulae (6.20) and (6.24) is that the RHS of 6.20 is expressed via the coframe $\vartheta$ in the original spacetime whereas the RHS of (6.24) is expressed via the coframe $\vartheta$ in the extended spacetime, see (4.2)-(4.4). In view of (4.10), (6.20) formula (6.24) can be rewritten as

$$
\mathbf{T}^{\mathrm{ax}}=T^{\mathrm{ax}}+\frac{4 m}{3} \vartheta^{1} \wedge \vartheta^{2} \wedge \boldsymbol{\vartheta}^{4} .
$$

The coordinate $x^{3}$ is redundant so $\mathbf{T}^{\text {ax }}$ can be viewed as a 3 -form in $(1+3)$ dimensional Lorentzian space with local coordinates $\left(x^{0}, x^{1}, x^{2}, x^{4}\right)$. Hence, we can define the covector

$$
\left(* \mathbf{T}^{\mathrm{ax}}\right)_{\boldsymbol{\delta}}:=\frac{1}{3 !} \sqrt{|\operatorname{det} g|}\left(\mathbf{T}^{\mathrm{ax}}\right)^{\boldsymbol{\alpha} \boldsymbol{\beta} \boldsymbol{\gamma}} \varepsilon_{\boldsymbol{\alpha} \boldsymbol{\beta} \boldsymbol{\gamma} \boldsymbol{\delta}}, \quad \boldsymbol{\delta}=0,1,2,4,
$$

which is the Hodge dual of $\mathbf{T}^{\mathrm{ax}}$. It is easy to see that we have

$$
\left\|\mathbf{T}^{\mathrm{ax}}\right\|^{2}=-\left\|* \mathbf{T}^{\mathrm{ax}}\right\|^{2} .
$$

Note that in the LHS of (6.27) we square a 3 -form in (1+4)-dimensional Lorentzian space whereas in the RHS of (6.27) we square a 1 -form in $(1+3)$-dimensional Lorentzian space, so we took great care in getting the sign right. Substituting (6.25) into (6.26) we get

$$
\left(* \mathbf{T}^{\mathrm{ax}}\right)_{\boldsymbol{\delta}}=\left(\begin{array}{c}
\frac{4 m}{3} \vartheta_{\delta}^{0} \\
* T^{\mathrm{ax}}
\end{array}\right)
$$


where $* T^{\mathrm{ax}}$ is the scalar defined by formula (6.21). It remains to observe that formulae (6.27), (6.28) imply 6.23).

The following theorem is the main result of our paper.

Theorem 6.2. In the special case with no dependence on $x^{3}$ a coframe $\vartheta$ and a density $\rho$ are a solution of the field equations for the Lagrangian density $L(\vartheta, \rho)$ if and only if the corresponding spinor field is a solution of the field equation for the Lagrangian density $L_{\mathrm{Dir}}^{+}(\xi)$ or the field equation for the Lagrangian density $L_{\mathrm{Dir}}^{-}(\xi)$.

Proof. Denote by $L(\xi)$ the Lagrangian density (4.8) but with $\vartheta$ and $\rho$ expressed via $\xi$. Accordingly, we rewrite the factorisation formula (6.7) as

$$
L(\xi)=-\frac{32 m}{9} \frac{L_{\mathrm{Dir}}^{+}(\xi) L_{\mathrm{Dir}}^{-}(\xi)}{L_{\mathrm{Dir}}^{+}(\xi)-L_{\mathrm{Dir}}^{-}(\xi)} .
$$

Observe also that the Dirac Lagrangian densities $L_{\text {Dir }}^{ \pm}$defined by formula (6.6) possess the property of scaling covariance:

$$
L_{\mathrm{Dir}}^{ \pm}\left(e^{h} \xi\right)=e^{2 h} L_{\mathrm{Dir}}^{ \pm}(\xi)
$$

where $h: M \rightarrow \mathbb{R}$ is an arbitrary scalar function.

We claim that the statement of the theorem follows from (6.29) and (6.30). The proof presented below is an abstract one and does not depend on the physical nature of the dynamical variable $\xi$, the only requirement being that it is an element of a vector space so that scaling makes sense.

Note that formulae (6.29) and (6.30) imply that the Lagrangian density $L$ possesses the property of scaling covariance, so all three of our Lagrangian densities, $L, L_{\text {Dir }}^{+}$and $L_{\text {Dir }}^{-}$, have this property. Note also that if $\xi$ is a solution of the field equation for some Lagrangian density $\mathcal{L}$ possessing the property of scaling covariance then $\mathcal{L}(\xi)=0$. Indeed, let us perform a scaling variation of our dynamical variable

$$
\xi \mapsto \xi+h \xi
$$

where $h: M \rightarrow \mathbb{R}$ is an arbitrary "small" scalar function with compact support. Then $0=\delta \int \mathcal{L}(\xi)=2 \int h \mathcal{L}(\xi)$ which holds for arbitrary $h$ only if $\mathcal{L}(\xi)=0$.

In the remainder of the proof the variations of $\xi$ are arbitrary and not necessarily of the scaling type (6.31).

Suppose that $\xi$ is a solution of the field equation for the Lagrangian density $L_{\text {Dir }}^{+}$. [The case when $\xi$ is a solution of the field equation for the Lagrangian density $L_{\text {Dir }}^{-}$is handled similarly.] Then $L_{\text {Dir }}^{+}(\xi)=0$ and, in view of (6.8), $L_{\text {Dir }}^{-}(\xi) \neq 0$. Varying $\xi$, we get

$$
\begin{aligned}
\delta \int L(\xi)=-\frac{32 m}{9}\left(\int \frac{L_{\mathrm{Dir}}^{-}(\xi)}{L_{\mathrm{Dir}}^{+}(\xi)-L_{\mathrm{Dir}}^{-}(\xi)} \delta L_{\mathrm{Dir}}^{+}(\xi)+\int L_{\mathrm{Dir}}^{+}(\xi) \delta \frac{L_{\mathrm{Dir}}^{-}(\xi)}{L_{\mathrm{Dir}}^{+}(\xi)-L_{\mathrm{Dir}}^{-}(\xi)}\right) \\
=\frac{32 m}{9} \int \delta L_{\text {Dir }}^{+}(\xi)=\frac{32 m}{9} \delta \int L_{\mathrm{Dir}}^{+}(\xi)
\end{aligned}
$$


SO

$$
\delta \int L(\xi)=\frac{32 m}{9} \delta \int L_{\text {Dir }}^{+}(\xi) .
$$

We assumed that $\xi$ is a solution of the field equation for the Lagrangian density $L_{\text {Dir }}^{+}$so $\delta \int L_{\text {Dir }}^{+}(\xi)=0$ and formula (6.32) implies that $\delta \int L(\xi)=0$. As the latter is true for an arbitrary variation of $\xi$ this means that $\xi$ is a solution of the field equation for the Lagrangian density $L$.

Suppose that $\xi$ is a solution of the field equation for the Lagrangian density $L$. Then $L(\xi)=0$ and formula 6.29 implies that either $L_{\text {Dir }}^{+}(\xi)=0$ or $L_{\text {Dir }}^{-}(\xi)=$ 0 ; note that in view of (6.8) we cannot have simultaneously $L_{\text {Dir }}^{+}(\xi)=0$ and $L_{\text {Dir }}^{-}(\xi)=0$. Assume for definiteness that $L_{\text {Dir }}^{+}(\xi)=0$. [The case when $L_{\text {Dir }}^{-}(\xi)=0$ is handled similarly.] Varying $\xi$ and repeating the argument from the previous paragraph we arrive at (6.32). We assumed that $\xi$ is a solution of the field equation for the Lagrangian density $L$ so $\delta \int L(\xi)=0$ and formula 6.32 implies that $\delta \int L_{\text {Dir }}^{+}(\xi)=0$. As the latter is true for an arbitrary variation of $\xi$ this means that $\xi$ is a solution of the field equation for the Lagrangian density $L_{\text {Dir }}^{+}$.

The proof of Theorem 6.2 presented above may appear to be non-rigorous but it can be easily recast in terms of explicitly written field equations.

\section{Discussion}

The mathematical model formulated in Section 4 is based on the idea that every point of spacetime can rotate and that rotations of different points are totally independent. The idea of studying such continua belongs to the Cosserat brothers [7]. Recall that in classical elasticity the deformation of a continuum is described by a (co)vector function $u$, the field of displacements, which is the dynamical variable (unknown quantity) in the system of equations. Displacements, of course, generate rotations: the infinitesimal rotation caused by a displacement field $u$ is $d u$, the exterior derivative of $u$. The Cosserat brothers' idea was to make rotations totally independent of displacements, so that the coframe (field of orthonormal bases attached to points of the continuum) becomes an additional dynamical variable.

Our model is a special case of Cosserat elasticity in that we model spacetime as a continuum which cannot experience displacements, only rotations. The idea of studying such continua is also not new: it lies at the heart of the theory of teleparallelism (= absolute parallelism), a subject promoted in the end of the 1920s by A. Einstein and É. Cartan 8, 9, 10. It is interesting that Einstein pursued this activity precisely with the aim of modelling the electron, but, unfortunately, without success.

The differences between our mathematical model formulated in Section 4 and mathematical models commonly used in teleparallelism are as follows.

- We assume the metric to be prescribed (fixed) whereas in teleparallelism it is traditional to view the metric as a dynamical variable. In other words, in works on teleparallelism it is customary to view (4.1) not as a constraint but 
as a definition of the metric and, consequently, to vary the coframe without any constraints at all. This is not surprising as most, if not all, authors who contributed to teleparallelism came to the subject from General Relativity.

- We choose a very particular Lagrangian density (4.8) containing only one irreducible piece of torsion (axial) whereas in teleparallelism it is traditional to choose a more general Lagrangian containing all three pieces (tensor, trace and axial), see formula (26) in 3 .

We now explain the motivation behind our choice of the Lagrangian density (4.8). Suppose for simplicity that we don't have electromagnetism, i.e. that $A=0$, in which case the extended coframe and extended metric agree (6.10). Let us perform a conformal rescaling of the extended coframe: $\boldsymbol{\vartheta}^{j} \mapsto e^{h} \boldsymbol{\vartheta}^{j}, j=0,1,2,3,4$, where $h: M \rightarrow \mathbb{R}$ is an arbitrary scalar function. Then the metric and axial torsion scale as $\mathbf{g} \mapsto e^{2 h} \mathbf{g}$ and

$$
\mathbf{T}^{\mathrm{ax}} \mapsto e^{2 h} \mathbf{T}^{\mathrm{ax}}
$$

respectively. Here the remarkable fact is that the derivatives of $h$ do not appear in formula (7.1) which means that axial torsion is the irreducible piece of torsion which is conformally covariant. It remains to note that if we scale the density $\rho$ as $\rho \mapsto e^{2 h} \rho$ then the Lagrangian density (4.8) will not change.

Thus, the guiding principle in our choice of the Lagrangian density (4.8) is conformal invariance. This does not, however, mean that our mathematical model formulated in Section 4 is conformally invariant: formula (4.4) does not allow for conformal rescalings. The Kaluza-Klein extension is a procedure which breaks conformal invariance, as one would expect when introducing mass.

The main result of our paper is Theorem 6.2 which establishes that in the special case with no dependence on $x^{3}$ our mathematical model is equivalent to the Dirac equation. This special case is known in literature as the Dirac equation in dimension $1+2$ and is in itself the subject of extensive research.

This leaves us with the question what can be said about the general case, when there is dependence on all spacetime coordinates $\left(x^{0}, x^{1}, x^{2}, x^{3}\right)$. In the general case our model is clearly not equivalent to the Dirac equation because it lacks one real dynamical degree of freedom, see last paragraph in Section 5 . Our plan for the future is to examine how much our model differs from the Dirac model in the general case. We plan to compare the two models by calculating energy spectra of the electron in a given stationary electromagnetic field, starting with the case of the Coulomb potential (hydrogen atom).

The spectral-theoretic analysis of our model will, however, pose a monumental analytic challenge. There are several fundamental issues that have to be dealt with.

- Our model does not appear to fit into the standard scheme of strongly hyperbolic systems of partial differential equations.

- The eigenvalue (= bound state) problem for our model is nonlinear.

- Our construction relies on the density $\rho$ being strictly positive. This assumption may fail when one seeks bound states other than the ground state. 


\section{References}

[1] V. Pasic and D. Vassiliev, PP-waves with torsion and metric-affine gravity Class. Quantum Grav. 22 (2005) 3961-3975.

[2] D. Vassiliev, Teleparallel model for the neutrino Phys. Rev. D 75 (2007) 025006.

[3] F. W. Hehl and Yu. N. Obukhov, Élie Cartan's torsion in geometry and in field theory, an essay Annales de la Fondation Louis de Broglie 32 (2007) 157-194.

[4] A. Dimakis and F. Müller-Hoissen, Solutions of the Einstein-Cartan-Dirac equations with vanishing energy-momentum tensor J. Math. Phys. 26 (1985) 1040-1048.

[5] A. Dimakis and F. Müller-Hoissen, On a gauge condition for orthonormal threeframes Phys. Lett. A 142 (1989) 73-74.

[6] A. Dimakis and F. Müller-Hoissen, Spinor fields and the positive energy theorm Class. Quantum Grav. 7 (1990) 283-295.

[7] E. Cosserat and F. Cosserat, Théorie des corps déformables, Librairie Scientifique A. Hermann et fils, Paris, 1909. Reprinted by Cornell University Library.

[8] R. Devever (editor), Elie Cartan and Albert Einstein: Letters on Absolute Parallelism, Princeton University Press, 1979.

[9] A. Unzicker and T. Case, Translation of Einstein's Attempt of a Unified Field Theory with Teleparallelism (2005), http://arxiv.org/abs/physics/0503046

[10] T. Sauer, Field equations in teleparallel spacetime: Einstein's Fernparallelismus approach toward unified field theory Historia Mathematica 33 (2006) 399-439.

James Burnett

Department of Mathematics and Department of Physics \& Astronomy

University College London

Gower Street

London WC1E 6BT

UK

e-mail: j.burnett@ucl.ac.uk

Olga Chervova

Department of Mathematics

University College London

Gower Street

London WC1E 6BT

UK

e-mail: olgac@math.ucl.ac.uk

Dmitri Vassiliev

Department of Mathematics and Institute of Origins

University College London

Gower Street

London WC1E 6BT

UK

e-mail: D.Vassiliev@ucl.ac.uk 